\title{
Design Alternatives for a MediaWiki to Support Collaborative Writing in Higher Education Classes
}

\author{
Sumonta Kasemvilas and Lorne Olfman \\ School of Information Systems and Technology, \\ Claremont Graduate University, Claremont, CA, USA
}

\author{
Sumonta.Kasemvilas@cgu.edu; Lorne.Olfman@cgu.edu
}

\begin{abstract}
Constructivist learning mechanisms such as collaborative writing have emerged as a result of the development of Web 2.0 technologies. We define the term mandatory collaborative writing to describe a writing activity where the group has a firm deadline. Our study focuses on how a wiki can fully support mandatory group writing. The motivation of this design science research study emerges from a graduate Knowledge Management class assignment to write a wiki book. The project outcome shows that the wiki instance used for the project, MediaWiki, could better facilitate the process with a set of extensions that support discussion, evaluation, and project management. We outline designs for these mechanisms: 1) a discussion mechanism that changes the way users discuss content on a wiki page and increases group awareness; 2) an evaluation mechanism that provides a tool for the instructor to monitor and assess students' performance; and 3) a project management tool that increases awareness of the status of each component of the writing project and provides an overall summary of the project. A demonstration of the principles to a focus group provided a basic proof of the validity of these mechanisms.
\end{abstract}

Keywords: awareness, collaborative writing, constructivist learning, design science research, discussion, evaluation, MediaWiki, project management, Web 2.0

\section{Introduction}

Wikis can be used to facilitate collaborative writing. Through the use of a wiki for a group writing project we discovered that some mechanisms can be added to wikis to support collaborative group writing in higher education. Collaborative writing can be used for varied purposes, which we term mandatory, optional, and hybrid. Based on the success of Wikipedia, it is generally assumed that a wiki is an effective collaborative writing tool. However, Wikipedia supports an optional collaborative writing purpose. Members of the Wikipedia community are voluntarily involved in a project that does not have deadlines or specific goals that the project needs to achieve. In contrast, a mandatory purpose in this context is one in which a group must finish its project

Material published as part of this publication, either on-line or in print, is copyrighted by the Informing Science Institute. Permission to make digital or paper copy of part or all of these works for personal or classroom use is granted without fee provided that the copies are not made or distributed for profit or commercial advantage AND that copies 1) bear this notice in full and 2) give the full citation on the first page. It is permissible to abstract these works so long as credit is given. To copy in all other cases or to republish or to post on a server or to redistribute to lists requires specific permission and payment of a fee. Contact Publisher@InformingScience.org to request redistribution permission. within a specific timeline. Examples of this type of collaborative writing are class projects and responses to requests for proposals. Both these purposes require a different project management style. In the mandatory case, project management must be formally applied or the deadline will not be met. In the optional case, there is no necessity for formal project management. A hybrid purpose, which has characteristics of the 
other two, is one where a project can be considered completed but with no real deadline. Examples of this hybrid purpose are a white paper being authored by a Community or Practice (CoP) and a set of requirements for an open source software project. We address mandatory collaborative writing in higher education in the paper.

Cunningham (2006) created the concept of a wiki. He specified a number of characteristics that he envisioned as necessary for enabling collaborative knowledge sharing. In this paper, we report on the problems encountered in using MediaWiki (one of the most popular wiki engines) in a classroom-based collaborative writing project. First, we review the literature on constructivist learning. Next, we compare the needs of the mandatory and optional purposes with the goals that Cunningham specified. Then, we provide a description of the course and its processes. We show that a wiki has the potential to support mandatory purposes, but requires additional facets to do so effectively. Next, we define the objectives for a solution and propose three mechanisms for MediaWiki to provide aid for discussion, evaluation, and project management functionality, and better support mandatory collaborative writing. The significant contribution of this study is these mechanisms, which can facilitate group writing and constructivist learning processes. We describe the design of these three mechanisms and explain how they support group writing and constructivist learning processes. Then, we conduct a preliminary demonstration and evaluation. The paper concludes with limitations and proposed plug-ins development and testing.

\section{Research Question}

Current wiki technology has several challenges in the way it supports collaborative writing, such as how group members delegate responsibilities, how they coordinate with each other, and how they evaluate the content of wiki pages. Therefore, we posed the following question: Can we design mechanisms to facilitate collaborative group writing in a classroom setting? To answer this question, we used our experience from a class that used MediaWiki as a mandatory collaborative writing tool using a design science methodology.

\section{Design Science Research}

According to Hevner, March, Park, and Ram (2004, p. 79), "design science addresses research through the building and evaluation of artifacts designed to meet the identified business need." This study follows six activities of design science research methodology (DSRM): 1) problem identification and motivation; 2) defining the objectives for a solution; 3 ) design and development; 4) demonstration; 5) evaluation; 6) communication (Peffers, Tuunanen, Rothenberger, \& Chatterjee, 2007). The purpose of the study is to design useful mechanisms for groups who are writing wiki pages, which need tools to support discussion and collaboration and help instructors with the assessment of collaborative student work. Researchers have designed a supporting library of tools and defined taxonomies to address some of these requirements, but these mechanisms are still in an evolving stage. A number of studies have attempted to solve the problems inherent in wiki technology, especially in Wikipedia (B. Suh, Chi, Kittur, \& Pendleton, 2008). This paper will focus on wiki collaborative writing among graduate students because collaborative writing is important in many graduate programs.

\section{Problem Identification and Motivation}

During the Fall 2006 semester, the second author of this paper, designed a graduate Knowledge Management course in which the goal was to write a textbook as the lone class assignment. The first author of this paper was one of the students in the class. The writing platform for the group writing project was a MediaWiki instance. In this section, we discuss the concept of constructivist learning that views learning as an active, social process. Given this approach, we suggest that group writing is an appropriate activity for facilitating constructivist learning. Then we justify the 
choice of using a wiki, and specifically, MediaWiki. Finally, we describe the details of the class project, and the outcomes of the project in terms of the quality of the book, some feedback from students as to their learning experiences, the effectiveness of MediaWiki for the task, and the technical problems discovered in writing process.

\section{Constructivist Learning}

Constructivism is a paradigm of learning. The most important concept in constructivism is a strong focus on student-centered learning. Constructivist learning theory considers the learner as a unique and complex individual who is actively involved in a collaborative learning process (Bruner, 1996; Dewey, 1916; Vygotsky, 1978). This theory views learning as an active, social process (Bruner, 1996; Dewey, 1916; Vygotsky, 1978). Thus, emerging knowledge is socially produced by the learner. In a constructivist learning environment, a student will actively participate with other students to learn together (Harasim, 1990). The learner is the focal point, as opposed to the lesson plan or subject matter, which the instructor will teach. Learning occurs in realworld settings, and not in prearranged settings constructed by the instructor. According to Dewey (1916), rather than passively receiving knowledge from the instructor, the active learner needs to do activities to construct knowledge. The instructor needs to provide reflective activities, such as case-based learning or hands-on experience to engage the minds of the learners in the learning process (Dewey, 1910). However, it is also important to the instructor to assure that the new information provided to learners is in an appropriate form and matches their level of understanding.

This theory also posits that learning takes place through social interaction and communication with others (Vygotsky, 1978). Success or failure depends on whether the learner feels more involved or hesitant to become closely involved with the group (Wegerif, 1998). The learner will construct knowledge by expressing ideas into words and sharing them with other learners and through negotiations among them. As learners may have different backgrounds and their own individual framework, when they collaborate with others, they can perceive a problem or a set of activities from various different perspectives. They might agree, share, explore, negotiate, and create meanings through shared understanding amongst their group. Responses and reactions to these ideas from other members, and group collaboration activities assist the student in constructing knowledge (Alavi, 1994; Bouton \& Garth, 1983). According to Hiltz, Coppola, Rotter, and Turoff (1999, p. 106), "learning is not only active but also interactive."

Thus, in the classroom setting, the role of the instructor is to lead and conduct discourse with real, challenging projects, allowing students to engage new information with their own knowledge construction schema through communication and collaboration with their classmates. The whole time, the instructor guides and helps students to refine their understanding until it matches with the meaning of the material. The students take responsibility for their own learning by developing their own metacognitive abilities to organize themselves in the learning process.

\section{Wiki Technology}

Cunningham created the first wiki in 1994 because he found that programmers had difficulties exchanging their ideas. Cunningham wanted to create a simple online database and let the community help each other to create contents. According to Cunningham (2006), "[a] wiki is a work made by a community" (p. 6). The original design of the wiki was geared so that everyone can edit whatever whenever they want if they feel like the content does not fit for them (Richardson, 2006). Some wiki implementers have added additional concepts to the original principles, which Cunningham created, including sharing, interaction, collaboration, and social network principles ("Wiki Design Principles," n.d.). Based on a recent white paper from the University of Delaware (2008) we assume that wiki technology can be used to support learning in higher education classes. The report introduces the idea of a Hybrid Wiki that includes features beyond those im- 
plemented by Cunningham. In the Hybrid Wiki, no users are anonymous, content is not made public, access is limited through various sets of permissions, copyright protections are maintained, contents include multimedia, contents are moderated by experts, and it there is a time limit for creating content.

Learning is not an immediate process. Learners need to revisit their understandings, contemplate on what they have learned, investigate their knowledge, and apply it. Learning is the process of going over one's own individual experiences and thoughts. Learners can take a long time to reach a moment of insight. Because of this, wiki technology is an appropriate technology to use in constructivist learning. Several features of wiki technology can support constructivist learning and collaborative writing. When learners gain new knowledge, they can come back to modify what they have previously written in a wiki. They can read what other users write, generate meaning through shared comprehension, refine and reshape their perceptions, and share or negotiate their understandings with other users.

Wikis can be used to assist teaching and learning (Raman, Ryan, \& Olfman, 2005; Parker \& Chao, 2007). However, because of the specific needs of instruction, assignments, and evaluation, classroom wikis need to be designed and used in a different way from Cunningham's original wiki design, which allowed anyone to edit a wiki however and whenever they pleased, and to do so anonymously. When we apply the wiki paradigm in a classroom environment, instructors have to formulate an evaluation model for fairness of individual and group assessments. Students need to finish group assignments based on instructors' requirements and within a limited timeframe. Students need to know their specific responsibilities, how they interact and collaborate with others, and their own progress as well as that of others. Instructors may need to act as "Editor-inChief" to manage scope, schedule, evaluation, and quality of contents (De PeDro et al., 2006). From this point of view, wikis that support mandatory collaborative writing cannot be open or arbitrary. Both students and instructors need to have specific roles and have to finish their tasks in a certain time period.

\section{Why MediaWiki}

MediaWiki was chosen as the platform for the class writing project because it is one of the world's most popular wikis- Wikipedia runs on MediaWiki - and it is easy to install, configure, and use. According to Barrett (2009), there are more than 2,000 wiki sites that use MediaWiki. These include Wikimedia projects such as Wiktionary, Wikisource, and Wikibooks. The option of moving the class project into the Wikibook space was also a motivation for choosing MediaWiki. Further, MediaWiki has a wide assortment of features, is very adaptable, and is scalable. It is a free and open source software platform, is very stable, has a mature development status, has an active community of developers, and can support 140 languages.

\section{Description of the KM Class Project}

Students taking a graduate class in knowledge management (KM) were required to write and edit an introductory textbook on the subject. This was the only assignment for the course. The class was composed of six males and three females. All students were familiar with using the Internet and some of them had used wikis before. The MediaWiki instance ran on a third-party server with restricted access. Students were motivated because the project was carried out in a class setting and their grades depended on their performance in writing and editing. Each student chose what major topics he or she wished to be responsible for writing in the KM book. The plan was for each student to finish one chapter of the book within the time limit of the semester. The "Main Page" of the book contained a detailed outline of the book chapters (see Figure 1). 


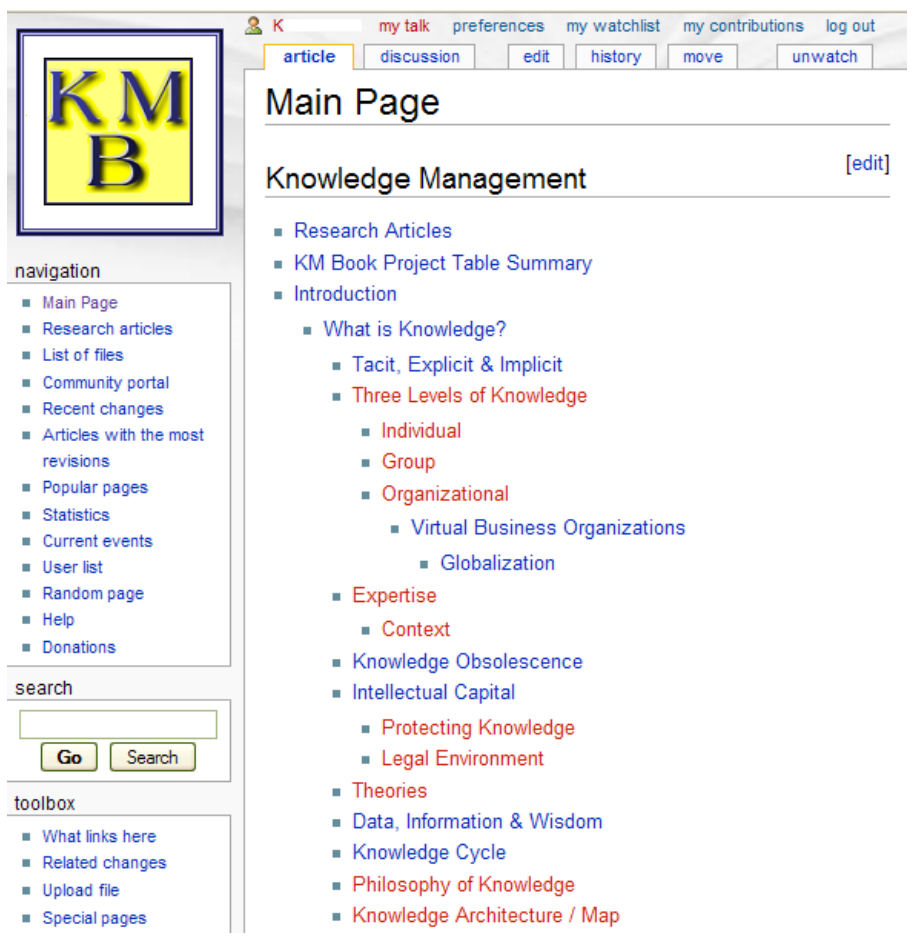

Figure 1: KM book main page

Editing roles were distributed across the group of nine students, with each student acting as Senior Editor each week and students in pairs acting as Associate Editors assigned to review 1/4 of the emerging content. The instructor acted as Editor-in-Chief. There were four roles (see Figure 2):

1. Writer: As a writer, a student was the first author on some pages of the book, and a secondary author on other pages. The pages each student contributed to as first author were ones that he or she expressed interest in writing about. Students could contribute to other pages as desired, with the possibility that his or her inputs would be first placed on the discussion page (in MediaWiki, there is a separate space for discussions of the content of pages) when it was not clear whether they fit closely with the writing that had already been contributed by the first author of that page. At times, the writer role and editor roles overlapped.

2. Editor-in-Chief: The Editor-in-Chief was the course instructor. He oversaw the writing process by working with the senior editors to assess the state of the book at the end of each week. He could make contributions as desired, but rarely did. The Editor-in-Chief made final decisions on debated issues, including ensuring that the book had a common look and feel. He discussed major decision items with the class members each week.

3. Senior Editor: The Senior Editor worked with the Editor-in-Chief and Associate Editors (pairs) to ensure the growth and development of the book. The Senior Editor ensured that the Associate Editors thoroughly edited their assigned portions of the book and provided an overall report of the book to the Editor-in-Chief. The Senior Editor asked the Editorin-Chief for advice on debated issues. The Senior Editor could make changes to pages, or make suggestions to revise and/or change pages. Suggestions were documented on the discussion pages. 
4. Associate Editor: Associate Editors worked in pairs to edit (carefully read, revise, and suggest additions and changes to) specific wiki pages. Working in pairs meant that students must find concurrent time to go over the sections of the book, which they were required to edit. Changes could be made directly on the wiki pages; suggestions had to be documented on the discussion pages. These may lead to debates about revisions.

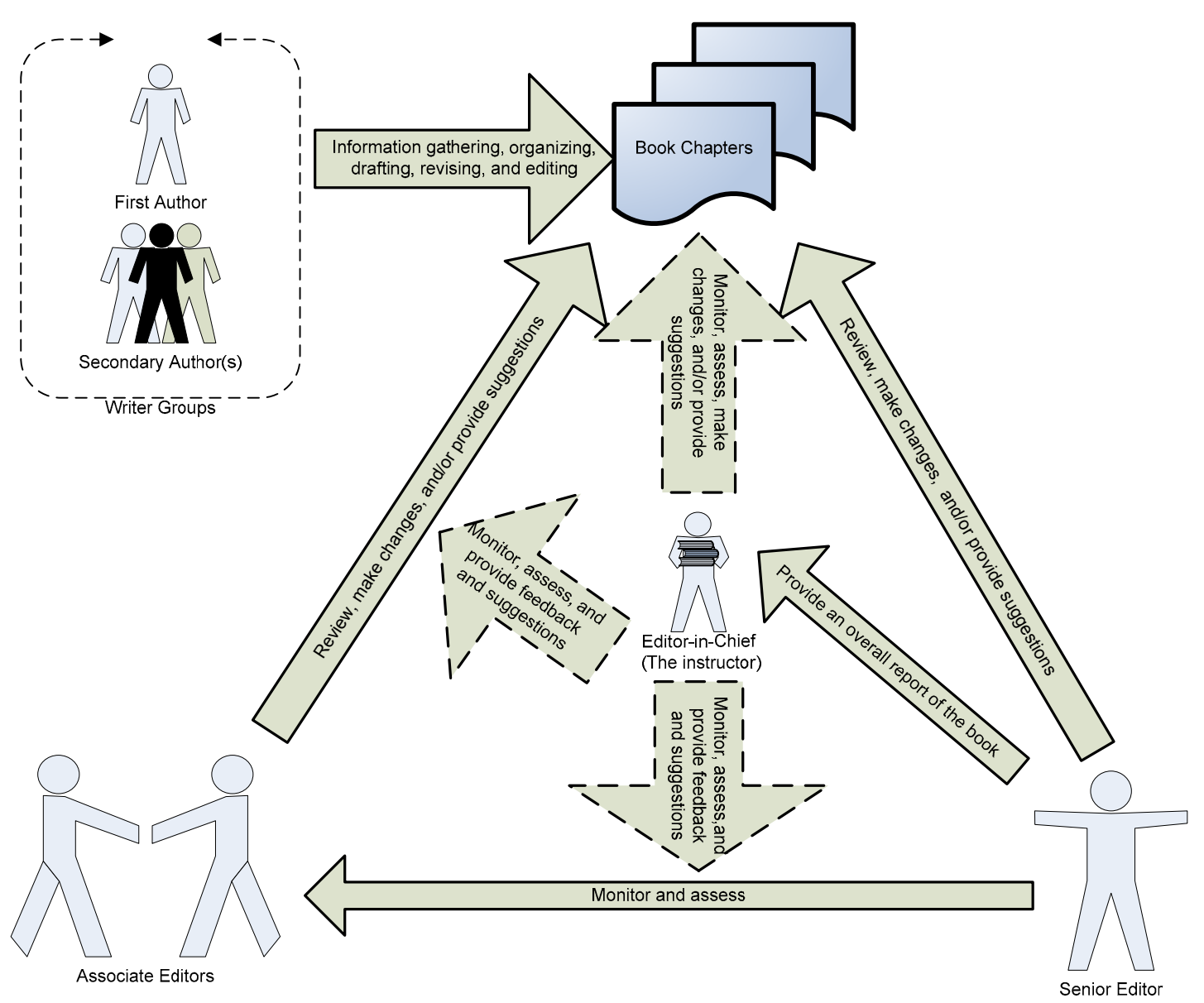

Figure 2: Model of collaborative writing in this study

While students were developing the content in the KM book, the Web site was open only for their use. The first few weeks of the class, the instructor provided a grade each week and feedback to students on what they should improve. The hope was that the students would produce good quality content, which could be made public after the class ended.

The instructor evaluated each student's contributed content and its quality. The instructor assessed students' performance by considering two rating categories for each page. First, information: the instructor evaluated what the page contributed to the subject it discussed, i.e., how the information in the page added to the detail of the book. The instructor used a 5-point scoring system to rate the quality of the information. A score of 3 meant that the information was basically not useful and could not be improved (it would have to be rewritten); a score of 7 meant that the information was minimally useful and could not be easily improved. A score 12 was allocated if the page needed further work but had the potential to contribute to the overall book content. A score of 15 meant that the page already had effective content but could be further improved; a 
score of 17 meant that the page was already publishable. Second, quality: the instructor evaluated the quality of each page and how ready it was for publishing. Quality was scored on a scale from 1 to 3 . A score of 1 meant that the page had many typos, formatting issues, etc. A score of 2 meant that page had a few typos and/or formatting issues. A score of 3 meant that the page had minimal typos and no formatting issues. The total score on each page was derived by adding the information score and the quality score. The overall range was 4 to 20. Additionally, the total score was factored by the number of words so that the greater the length, the higher the overall information score. The instructor also considered the priority of the primary author. A student who was the primary author of information would get $100 \%$ credit if there was no secondary author in that topic. If a student was a primary author and there were other authors in that topic, the primary author got $80 \%$ of the full score, while other students who contributed to existing content shared the remaining $20 \%$.

\section{Project Outcome}

As noted above, each of the nine students was responsible for writing about one of nine major topics. The amount of writing is summarized in Table 1 . There were 90 pages created in the wiki, with a total of 40,633 words (including references). More than half the pages received a total score of 19 or 20, showing that the students did a highly credible job of writing. The work of two students accounted for almost $50 \%$ of the total factored score (information, words, quality and primary authorship being the factors that were used for this calculation).

\begin{tabular}{|l|r|}
\hline \multicolumn{2}{|c|}{ Table 1: The results of KM book project } \\
\hline \multicolumn{1}{|c|}{ Project Outcome } & \multicolumn{1}{|c|}{ Value } \\
\hline N & 90 \\
\hline No. of pages in the wiki & 40,633 \\
\hline Average information score & 15.88 \\
\hline Average quality score & 2.21 \\
\hline No. of words per page & 452 \\
\hline Average & 518 \\
\hline SD & 17 \\
\hline Minimum & 3,650 \\
\hline Maximum & 280 \\
\hline Median & \\
\hline
\end{tabular}

Course evaluations showed that the students were less than "somewhat satisfied" with the organization of the course and the amount they learned in the course. The ratings were lower than normal for the instructor. One concern that emerged from written comments was that it is difficult to write a text about a topic area where students have no previous knowledge. Another key problem was that it was difficult for the instructor to provide interim grades throughout the course, especially with respect to the editing. The instructor was reluctant to give too much feedback to writers because he wanted the students to exert their editorial roles. However, the editorial effort was often "hit and miss". Moreover, over time, it became more difficult for everyone to manage the 
writing process. MediaWiki does not support threaded discussion. Over time, students in the class found it increasingly difficult to post their comments and discuss the content of a wiki page in its discussion page. The students sensed a lack of control and accountability in such a collaborative setting. The first author realized that design opportunities emerged from these difficulties.

\section{Problems Discovered}

Many difficulties emerged in using MediaWiki for this project:

1. Watchlists are a feature of MediaWiki to keep track of pages that are interesting to a user. Whenever these page change, the system will notify the user of the changes. However, this still requires the user to discover the specific changes that have been made. If they wanted to comment on changes to a page, they would use the Discussion page (see Figure 3 ). Keeping track of changes to discussion pages added another layer of complexity.

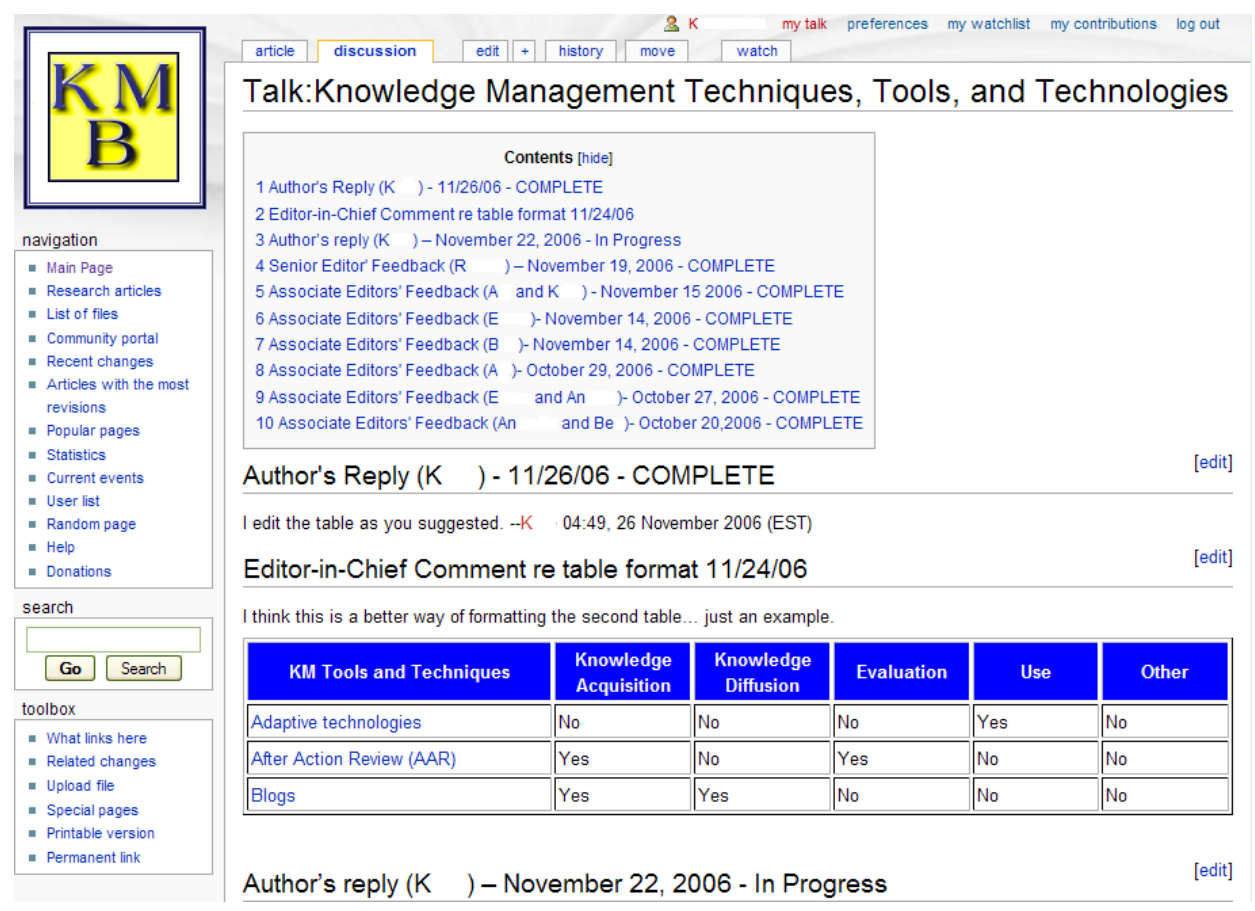

Figure 3: KM book discussion page

2. The first author built a table (page) to overview the status of the entire project (see Figure 4). It became clear that it would be very useful if an entry in the "Discussion" page could automatically update the summary table. The table showed the status of a particular (group-authored) page as either "ready for writing", "ready for editing", "complete", or "not started". The "ready for writing" status would be set by an Editor who has completed an analysis of the page. The "ready for editing" status would be set by a writer who has completed edits to a page. It was decided that editorial notes should be placed last-infirst-out in the "Discussion" page. Notes were written alternatively by an Editor and a Writer. The type of note had a particular "heading" characteristic that would annotate the required changes or summarize recent changes.

3. While the summary table was useful, we also needed a mechanism to automatically populate and update it. For example, anyone who was interested in being the main editor of a specific topic in the KM book would start writing a page, and the mechanism would up- 
date the summary page by putting that person's name in the main author field, updating the status of that page whenever he or she makes some progress. Thus, the Senior Editor could start his or her work from this table before going into details of each topic.

4. Some issues with the writing process were organizational (e.g., maintaining motivation, developing formatting standards, determining protocols for work status, etc.) and could not be solved by adding technical mechanisms to the wiki.
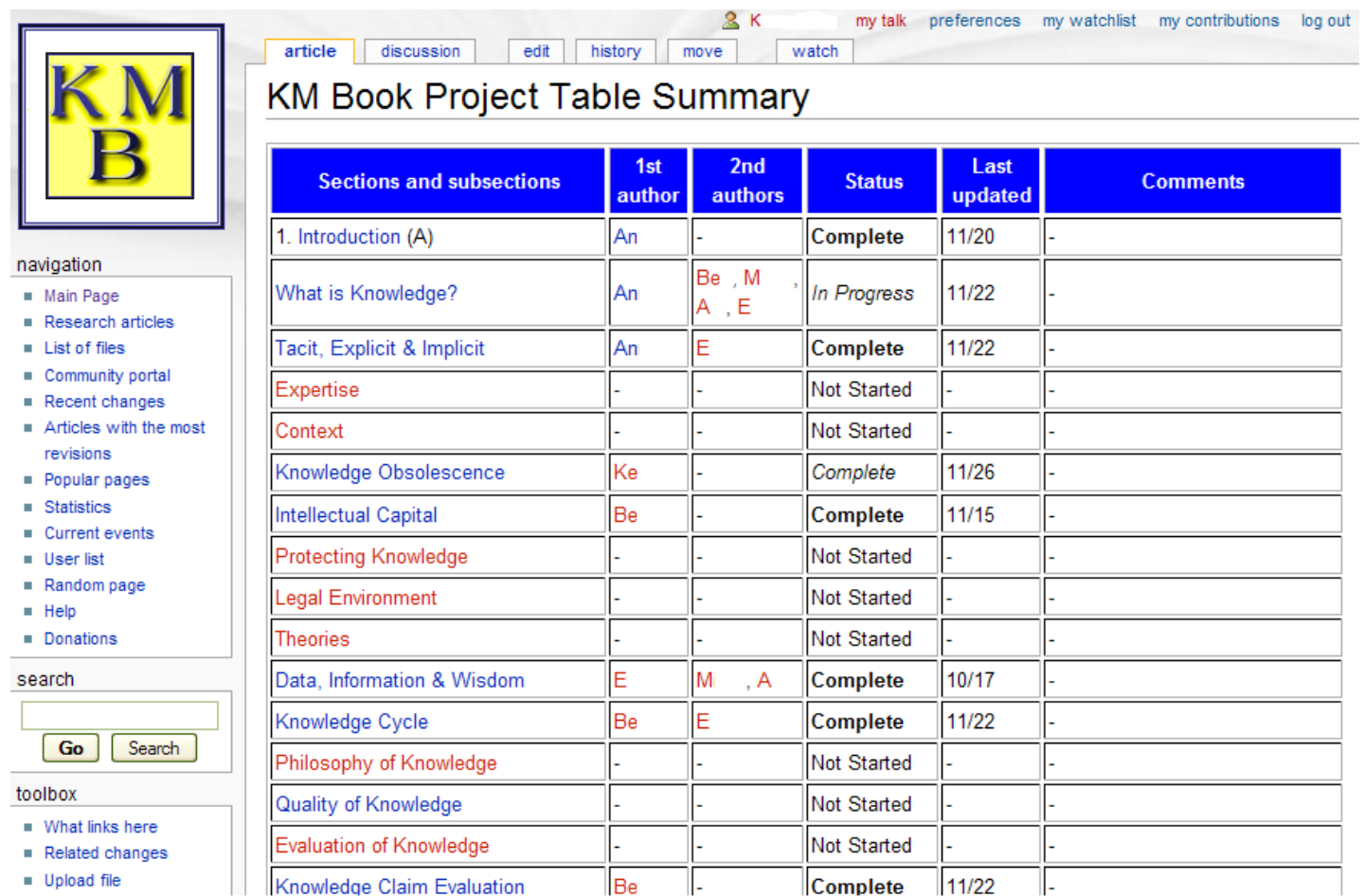

KM Book Project Table Summary

\begin{tabular}{|c|c|c|c|c|c|}
\hline Sections and subsections & $\begin{array}{c}\text { 1st } \\
\text { author }\end{array}$ & $\begin{array}{c}\text { 2nd } \\
\text { authors }\end{array}$ & Status & $\begin{array}{c}\text { Last } \\
\text { updated }\end{array}$ & Comments \\
\hline 1. Introduction (A) & An & - & Complete & $11 / 20$ & - \\
\hline What is Knowledge? & An & $\begin{array}{l}\mathrm{Be}, \mathrm{M} \\
\mathrm{A}, \mathrm{E}\end{array}$ & In Progress & $11 / 22$ & \\
\hline Tacit, Explicit \& Implicit & An & E & Complete & $11 / 22$ & - \\
\hline Expertise & - & - & Not Started & - & - \\
\hline Context & - & - & Not Started & - & - \\
\hline Knowledge Obsolescence & $\mathrm{Ke}$ & - & Complete & $11 / 26$ & - \\
\hline Intellectual Capital & $\mathrm{Be}$ & - & Complete & $11 / 15$ & - \\
\hline Protecting Knowledge & - & - & Not Started & - & - \\
\hline Legal Environment & - & - & Not Started & - & - \\
\hline Theories & - & - & Not Started & - & - \\
\hline Data, Information \& Wisdom & $E$ & M , A & Complete & $10 / 17$ & - \\
\hline Knowledge Cycle & $\mathrm{Be}$ & $\mathrm{E}$ & Complete & $11 / 22$ & - \\
\hline Philosophy of Knowledge & - & - & Not Started & - & - \\
\hline Quality of Knowledge & - & - & Not Started & - & 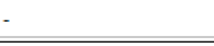 \\
\hline Evaluation of Knowledge & - & - & Not Started & - & $L^{-}$ \\
\hline Knowledge Claim Evaluation & $\mathrm{Be}$ & F & Complete & $\longdiv { 1 1 / 2 2 }$ & - \\
\hline
\end{tabular}

Figure 4: KM book project table summary

\section{Define Objectives of a Solution}

The focus of this paper is on the design of solutions to the weaknesses of MediaWiki when used as a group writing tool in a classroom setting. As a result of our experiences in the group writing activities described above, the objective was to design mechanisms to support some requirements of collaborative writing in higher education. Solutions to organizational problems require a redesign of the course process and are not addressed here. In this section we propose some solutions to the problems the class faced in performing activities such as commenting, evaluation, and project management. We first discuss principles of group (collaborative) writing, outline other research and solutions related to these processes, and then specify requirements that can lead to improving these processes.

\section{Group (Collaborative) Writing in the Classroom}

According to Tammaro, Mosier, Goodwin, and Spitz (1997), "Writing is a complex process" (p. 21). Although the complexity and difficulty of writing activities increase dramatically when more than one person is involved, there are many advantages to group collaboration, such as encouraging group members to work in cooperative ways to share knowledge and exchange ideas. As predicted by constructivist learning theory, group collaboration can overcome limitations of individual knowledge and skills (McCarthy, Miles, \& Monk, 1991). Individuals can create new content 
individually or with other group members, and help each other improve quality. Feedback from instructors is another factor that can encourage students to perform better (Barros \& Felisa Verdejo, 2000; H.-J. Suh \& Lee, 2006). However, the disadvantages of group collaboration are the difficulty of coordinating a joint effort and the diversity of group members (McCarthy et al., 1991). In collaborative writing, it is difficult for members to follow the discussion and add their own points (Weng \& Gennari, 2004). Instructors also find it difficult to monitor, assess, and promote students' contributions and performance (H.-J. Suh \& Lee, 2006).

Posner and Baecker (1992) developed a taxonomy that categorized roles, activities, control methods, and strategies in a collaborative writing project. They identified that each person in the group has at least one role: writer, consultant, editor, and reviewer. Collaborative writing is composed of several sub-activities: brainstorming, note taking, organizational planning, writing, revising, and editing. Posner and Baecker (1992) categorized control methods: centralized (only one person controls all documents in the project), relay (one person controls all documents at a specific time and control can pass among group members), independent (each person has responsibility for a separate part of the document and controls his or her own part), and shared (several group members have equal privileges to control the document at the same time). They also classified strategies of a collaborative writing project: single writer (one person writes the document but he or she may get the ideas from discussion with other members), scribe (only one person has the responsibility of writing the group's thoughts), separate writers (group members split the document into parts and each person takes responsibility for an individual part), and joint writing (group members collaborate and decide the content of the document and then write the document together).

The writing process is not static while the document is evolving, especially in a group setting. Beck and Bellotti (1993) conducted interviews, surveys and case studies to analyze the coauthoring process. They concluded that the nature of a group is dynamic. Roles, goals, activities and strategies change over time as group members respond to external influences, which change circumstances and bring unpredicted events.

In higher education, collaboration is vital. Graduate students need to perform collaborative assignments, do research and case studies, as well as review, summarize, and critique papers. There are many ways to manage how students will perform collaborative writing. For example, Schulz and Ludlow (1996) developed group writing models where the instructor allowed students to divide the topic into sections and let each student in the group individually take responsibility for finishing his or her own section. Another model is where the instructor singles out one student in the class to start writing on the assigned topic, while the other students in the group proofread and edit the content. It is vital for the instructors to design what model of group writing they wish to use and clearly inform students.

As can be seen from this review, the design of the pedagogy for a group writing class entails many choices by the instructor. It is not clear from the literature which choices to make. Moreover, the technical issues associated with facilitating the group writing process are not addressed by the literature.

\section{Related Research and Proposed Solutions}

Constructivism plays a vital part in defining how wikis can be used for collaborative writing. Ideally, the technology (e.g., a wiki) should not inhibit group writing activities. Our experience indicated that it is necessary to improve discussion procedures since this is a crucial part in collaboration. From a technical standpoint, the students found it difficult to follow the discussion and add their own points because in MediaWiki, the content page and discussion page are separated. This also relates to workspace awareness (Liccardi, Davis, \& White, 2007, 2008a, 2008b). As the 
amount of content increases, members may find it is difficult to locate the section in the content that they want to discuss. Kriplean, Beschastnikh, McDonald, and Golder (2007) assert that MediaWiki needs a mechanism to provide an annotated version of conversations to keep better track of discussions: which participants were involved in the process, what had been discussed so far, and where to locate updates in the conversation on the discussion page. Designing a mechanism to improve the discussion page would be beneficial for group members in a constructivist learning environment, so that they can easily comment, reply, argue, create threads of conversations, categorize discussions, keep track of content, and involve their peers in the decision and negotiation process. In this way, students can be at the center of the learning process, and will be able to more easily monitor and direct their ability to construct knowledge.

One dominant characteristic of constructivism is learning as a social activity. Learning and cooperation involve an intimate connection to other group members. Knowing what activities one's peers are engaging in while writing in the project provides awareness in social aspects by supporting conversation and interaction with other members. According to Kriplean et al. (2007), MediaWiki needs an awareness tool, which can summarize activities to better assist the agreement process among wiki members. Several studies have attempted to improve wiki workspace awareness and users' awareness to enhance the effectiveness of collaborative writing. One example is CAWS (Co-Authoring Wiki based System) (Liccardi, 2007; Liccardi et al., 2007, 2008a, 2008b), which aims to design co-authoring features in wiki-based systems to support the collaborative authoring system and increase awareness. CAWS focuses on four aspects of awareness: personal, social, informal, and group (Liccardi et al., 2008a). CAWS also provides an annotation system attached to threaded discussions, and users can filter comments by type or by author (Liccardi et al., 2008b). CAWS is in a stable stage, but it still lacks other functionality, such as instant messaging and a polling system (Liccardi et al., 2008b). While CAWS is based on the wiki concept, it is not implemented on the MediaWiki platform.

Instructors also find it difficult to assess students' participation and performance in wiki usage (Forte, 2006; Forte \& Bruckman, 2007). Forte (2006) and Forte and Bruckman (2007) are designing and developing wiki tools, which assist instructors in assessing students' participation. Motivation is one of the most important keys to learning. It is not only assists the learning process, but is also indispensable in learning. According to Bandura and Cervone (1983), the goals of individuals are not the sole factor that affects change in the motivation level of individuals: goals must be combined with performance feedback of progress towards meeting or accomplishing them. Instructors are the ones who can provide feedback to engage students in the learning process. Students have the goal of receiving a good grade in the class. From a constructivist perspective, the instructor is the mentor who guides and provides support to students. Yet, it might be helpful if a system can provide feedback to automatically in order to encourage students to better engage or maintain their efforts in the learning environment.

Collaborative writing may require a project management feature, which was one of the missing elements in MediaWiki. Student feedback in the course evaluations revealed declining motivation and action over time, a general sense of lack of control and accountability in the collaborative setting, and a lack of clarity in direction. All of these are useful points to justify why a project management feature would be useful. Additionally, from a constructivism perspective, this mechanism provides awareness and motivation to students. They can see how much progress they have made when compared with their peers.

\section{Design Alternatives for MediaWiki Extensions}

We propose three mechanisms that could be developed as extensions to MediaWiki: a discussion mechanism for supporting an effective asynchronous discussion, an evaluation mechanism for 
assisting instructors to provide feedback and evaluate students' contributions, and a project management mechanism for guiding students through the writing process.

\section{Discussion Mechanism}

Weng and Gennari (2004) proposed an asynchronous collaborative writing system through annotations. We apply their model to design a discussion mechanism for MediaWiki. The discussion mechanism model (Figure 5) has two layers, interface and content. In the content layer, the evolving wiki document is composed of three contexts: page, text, and annotation. Students and instructors utilize the Discussion Tool in the interface layer to add threaded discussion or annotation in the wiki document. The activities from students and instructors are kept in the Discussion Database. The Awareness Tool calls the stored procedure in the Discussion Database to retrieve information from the Discussion Database and notify instructors and students based on different filters, such as annotations made by each user, annotation category, and newly-added annotations in the specified period of time. The Discussion Database collects context (metadata of the content that an annotation is attached to, i.e., the page, text, and annotation context), message body (the actual text of the annotation), annotation creator, annotation recipient, annotation time and date, response (used in threaded discussions), status (what happened to a specific annotation), category (the annotation type, such as reply or comment), voting (most dissatisfied to most satisfied), and urgency (the priority of annotations) (Weng \& Gennari, 2004).

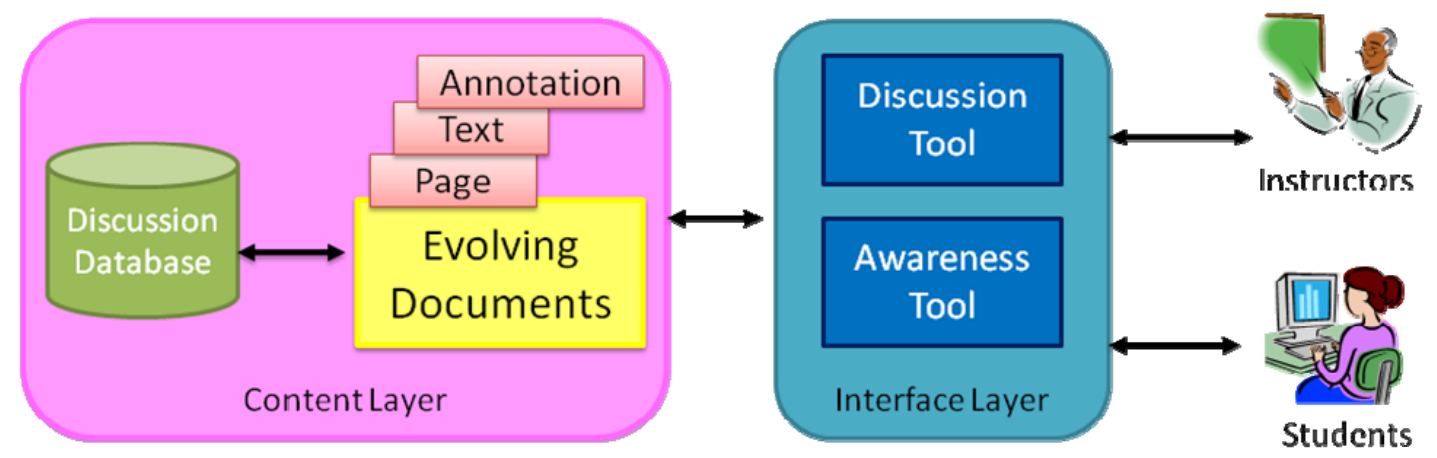

Figure 5: Discussion mechanism (Adapted from Weng and Gennari, 2004)

As noted above, MediaWiki does not have a good discussion system. Users have to go to a discussion page to add comments. These comments can be placed at any section of the page, and are not left unsorted. The more students involved in a discussion, the more comments are posted, and it quickly becomes difficult to track and organize comments. Also, without an awareness system, it is difficult for users to identify what has been changed, who has made the changes, and when those changes were made. Our proposed mechanism will provide a discussion area on the same page with the wiki content page. The process could work as follows:

A student starts the first discussion thread by highlighting a paragraph, selecting what category this thread should be sorted under (i.e., suggestion, agreement, disagreement), and designating the importance of the thread. Other students can reply to this discussion, and rate each comment (by approval or importance). Those who contribute to the discussion thread have the ability to edit or delete their content. Whenever anyone creates or replies to a discussion thread, the system will update the data in the database and notify all participants. Students can filter the discussions by category (i.e., username, date, type) and therefore maintain their own level of awareness. 


\section{Evaluation Mechanism}

Barros and Felisa Verdejo (2000) proposed a model to improve group awareness and group performance. In this paper, we apply their model to design an evaluation mechanism in MediaWiki (Figure 6). The Performance Database that we will incorporate into MediaWiki will record all the accesses and the actions performed by users (e.g., user identification, time and date, host computer, wiki page (workspace), activity, task, and type of action ) when they edit a wiki content page, including adding comments and discussions from the Discussion Database. The Quantitative Analysis Tool queries a variety of parameters such as the number of student contributions during a period, the number of hourly accesses for a group in an activity, the number of contributions by each student, the number of contributions by each student for all the group members in an activity, contributions of each group to each task, the number of contributions by students for an activity, and the evaluation of each discussion of a task, to display when users request it (Barros \& Felisa Verdejo, 2000). The output of the Quantitative Analysis is information on students' participation.

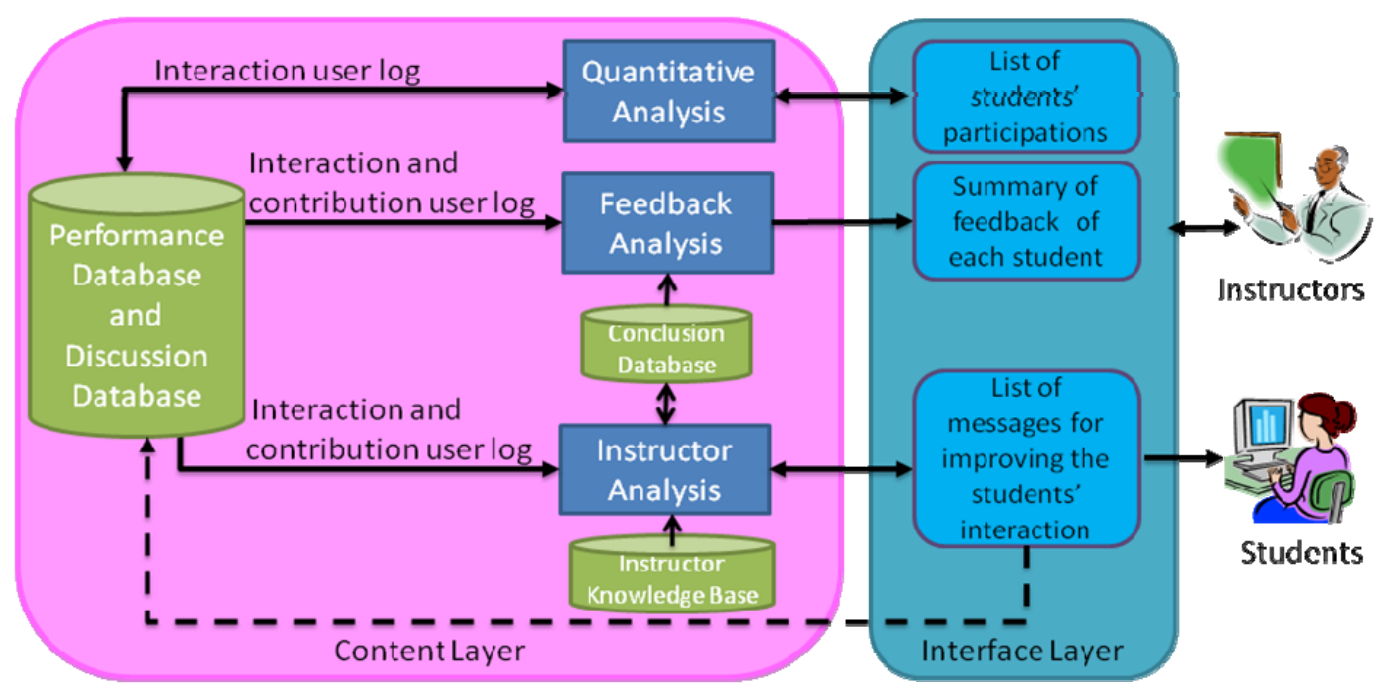

Figure 6: Evaluation mechanism (Adapted from Barros and Felisa Verdejo, 2000)

The Instructor Analysis Tool uses simple inference rules to generate messages following the pattern "if attribute-value then message." The Instructor Knowledge Base is composed of the definitions of the attributes of the sets and the rules relating to them. It can include the number of contributions that each student added, the mean size of a student's contributions, the number of contributions authored by others that have been answered by this student, and the number of contributions authored by the student that were continued or answered (Barros \& Felisa Verdejo, 2000). The Instructor Analysis Tool uses the results of the Performance Database as input and generates feedback messages based on students' performances. The instructor can modify the message before sending it. The Feedback Analysis Tool is used for improving group interaction. This module examines students' contributions before and after the instructor sends out a message. It retrieves data from the Conclusion Database to summarize feedback on each student to help instructors judge whether the feedback has been appropriate and increases students' participation.

\section{Project Management Mechanism}

According to Bean and Hott (2005), there are some wiki providers, such as Socialtext (http://www.socialtext.com) and JotSpot (http://jot.com) that have project management features. 
These leading enterprise wiki vendors provide an extensive, complex system for enterprise wikis, which have license fees. Suh et al. (2008) proposed two types of WikiDashboard - Article Dashboard and User Dashboard - for live Wikipedia pages. The WikiDashboard provides a graph that presents information about how many revisions have been made on each Wikipedia page and who edits this Wikipedia page. This WikiDashboard displays on the top of each Wikipedia page, which allows users to easily assess the status of a page.

The project management mechanism (Figure 7) retrieves an interaction user log from the Performance and Discussion Databases to create a Project Status Summary Database. The mechanism retrieves data from the Project Status Summary Database to display the status of each page on the top of the wiki page and automatically updates the table summary of the project. In the summary page a table displays an overall picture of the project such as who the first author is, who the second authors are, when last modified and by whom, as well as current status of this page (i.e., not started, being edited, updated on, not yet updated, requires more review, and completed).

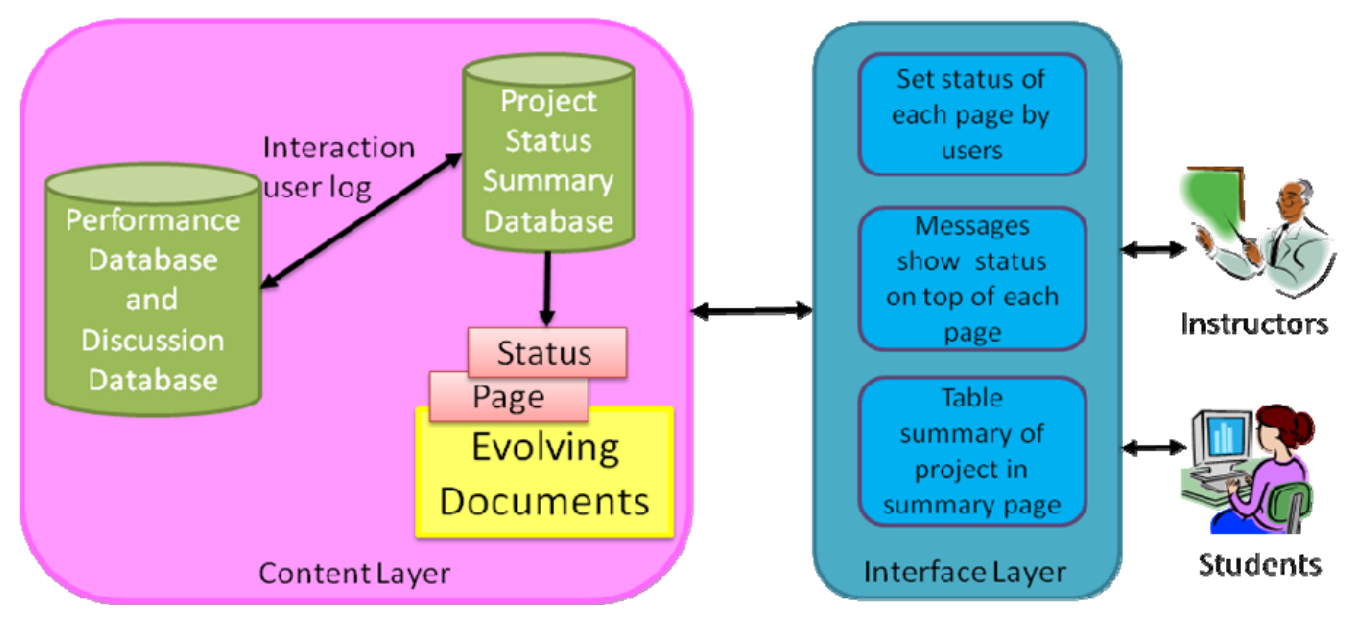

Figure 7: Project management mechanism

Authors and editors can modify the status of each page (e.g., ready for writing or ready for editing) and it is recorded in the Project Status Summary Database. They can make changes to finalize the status of the page, but we limit changing status only for authors. The Editor can suggest or discuss the page in the Discussion Tool based on the status, which is shown on the top of the page.

\section{Preliminary Demonstration and Evaluation}

According to Hevner et al. (2004), design science research is composed of an iterative build-andevaluate loop. We did a preliminary demonstration and evaluation of our designs by discussing a preliminary project management mechanism with the KM class, and by conducting a focus group in a doctoral seminar during the 2008 spring semester.

\section{Demonstration}

The model of the project management mechanism was the one shown in Figure 4. We demonstrated the model of our discussion and evaluation mechanism designs to the participants in the focus group, and explained how they work. Then we provided examples of a variety of user interface prototypes to them: collaborative protocol reviewing with an awareness system (Weng, Gen- 
nari, \& McDonald, 2004) (Figure 8); CommentPress ("The Institute for the Future of the Book," n.d.) (Figure 9), which provided an innovative interface with a threaded discussion; and a mouse over interface, which displays a dialog box from which users could see and add comments directly in the box (Figure 10). We explained how each of these prototypes function. We also showed how the current discussion page of MediaWiki works because they were not using discussion pages.

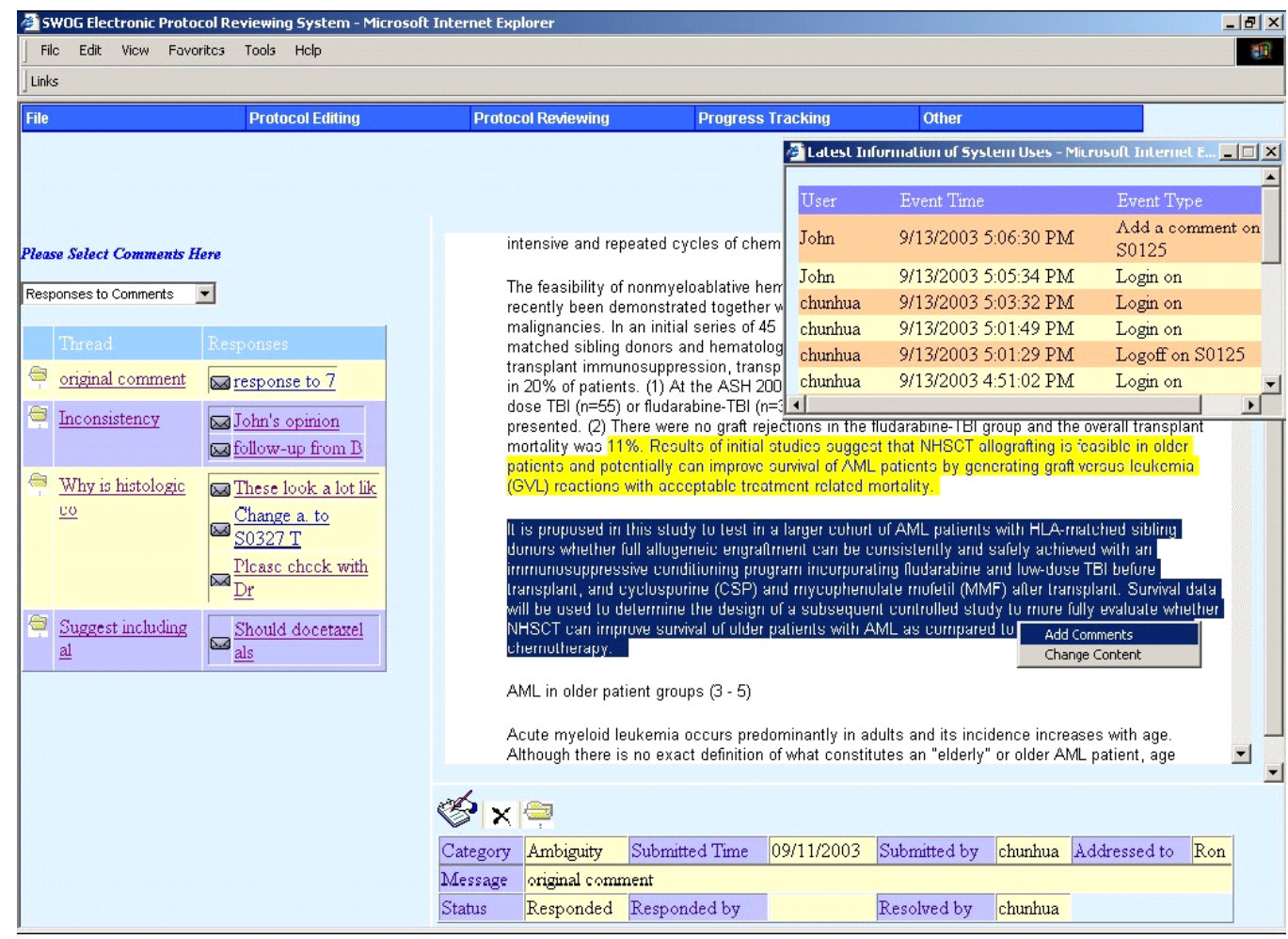

Figure 8: Collaborative protocol reviewing (Used with permission from Weng, Gennari, and McDonald, 2004)

\section{Evaluation}

For the project management mechanism, we demonstrated and evaluated this design in the KM class. After we built the summary table, students in the class provided positive feedback and agreed that this was a useful tool in tracking the overview of the KM book.

For the discussion mechanism, students liked the various designs because they provided an easier way to keep track of what they and their peers have done. The students suggested a way to track changes made by different authors, like the Track Changes feature in Microsoft Word. Some students suggested a private space to keep drafts before posting them in the public space. Further, some students wanted the ability to comment privately. One student liked the mouse-over interface, but also liked the idea of displaying the topic of each section as in CommentPress.

Based on this evaluation, we found that our proposed design would be useful to students in graduate courses. Most participants preferred a simple user interface, which provided enough information for them to use in a discussion. They also preferred the evaluation mechanism, which provided automatic feedback to them.

All participants in our focus groups have given us positive feedback and suggested ideas to improve the design of the interface. Based on the feedback that we received, our next step is to de- 
velop the prototype based on our proposed design, conduct a usability test and test the efficiency of the first prototype, assess whether it is appropriate in a classroom setting, and gather feedback to redesign and improve the mechanisms.

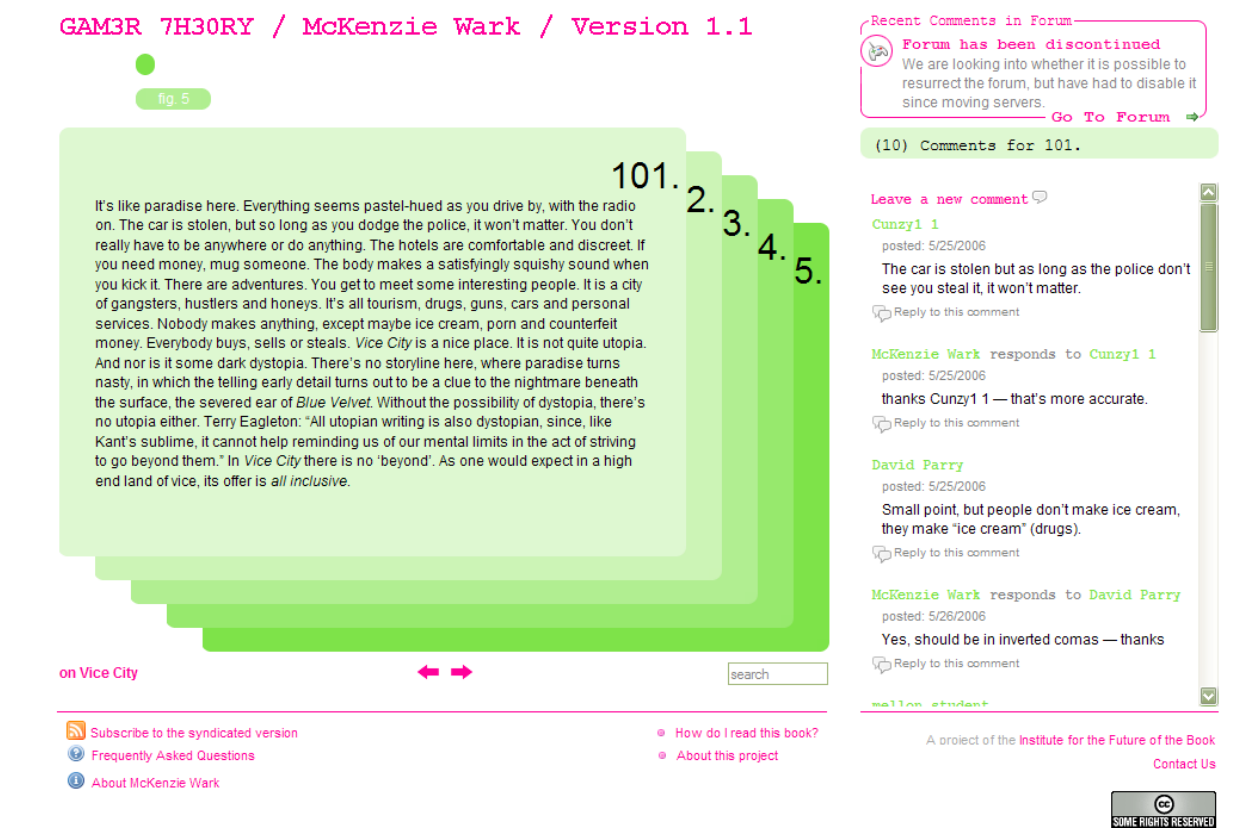

Figure 9: CommentPress in Gamer Theory project (Used with permission from "The Institute for the Future of the Book," n.d.)

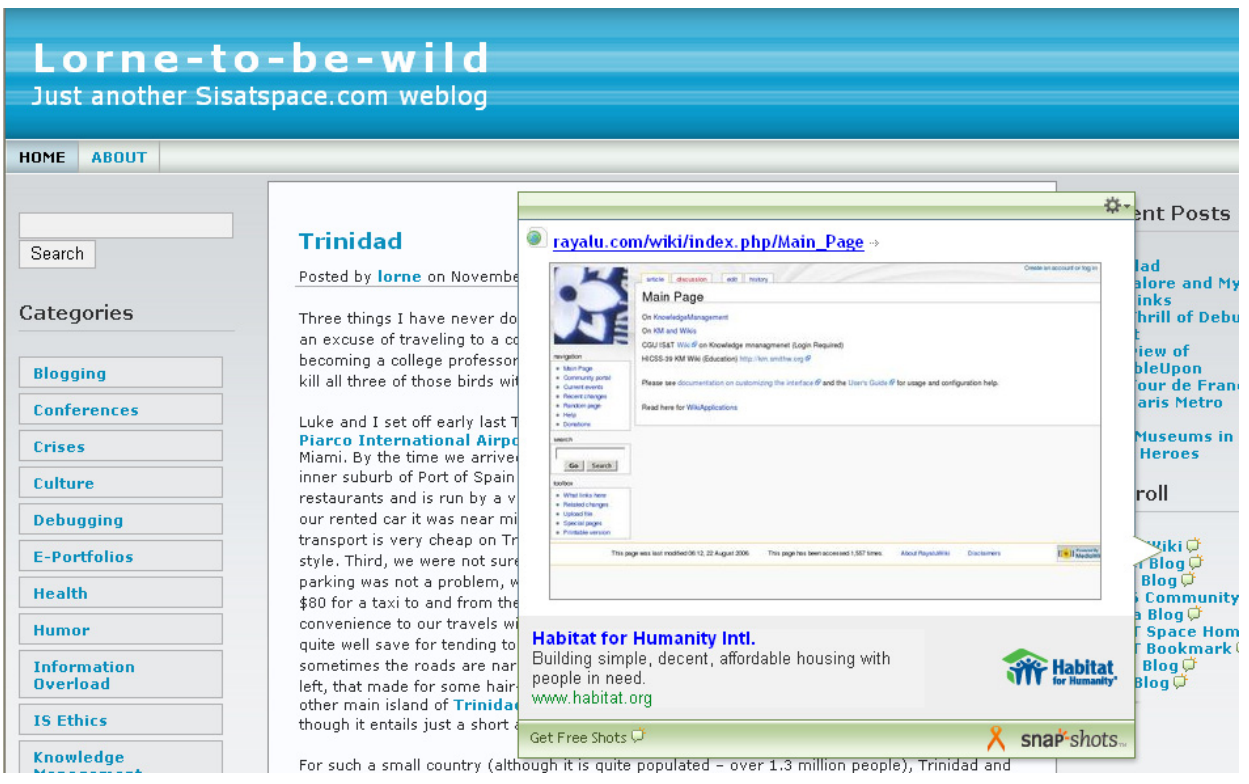

Figure 10: An example of mouse over interface 


\section{Conclusion and Future Work}

MediaWiki is a useful tool for supporting group collaboration but when we apply it to the academic setting, we need to consider and adapt some features to match the needs of the classroom environment, which requires mandatory collaborative writing. The major success factor of using wikis in collaborative work is the motivation of members. Wiki technology can promote the collaboration environment and enhance communication and knowledge sharing; however, the vital part originates from group members themselves who have the responsibility to achieve the goals of mandatory collaboration in group writing. With assistance from appropriate planning and organizing, using wikis for collaborative writing can produce effective learning outcomes with the addition of some new tools.

One limitation of this research is that it addresses only one wiki instance, MediaWiki. TWiki and TikiWiki provide threaded discussions instead of discussion pages. TWiki is more appropriate for small-medium businesses or large enterprises that need a full, complex system and can pay for subscriptions to support it, meanwhile TikiWiki is more appropriate for one who needs the full features of a Content Management System (CMS), including a discussion forum and blog. TWiki also provides project management features, and is more suited as a project management tool to be used in organizational settings, and not for group writing in a small classroom setting. Other wiki software platforms, such as Wikispaces and PBWiki are not open source, and cannot be modified to tailor the system to our specific demands.

Another limitation of this research is in fulfilling some of the requirements of DSRM. It is not a complete design, as it is only iteration. It lacks a complete evaluation. We plan to build the proposed plug-in prototypes and evaluate their effectiveness and usability over the next six months. According to Hevner et al. (2004), the nature of good design is iterative. The process (set of activities) of creating the product (an artifact) that can address a business need and solve a problem is usually to go back to the design stage again and again after the designed artifact is tested, used, and observed. Further study will be needed to redesign better mechanisms.

This study follows the DSRM process model for Information Systems research (Peffers et al., 2007). Problems were surfaced as a result of using a wiki to support collaborative writing on a mandatory basis. In this paper, we propose three mechanisms designed to support collaborative writing in higher education systems using MediaWiki: 1) a discussion mechanism based on Weng and Gennari (2004), which changes the way people discuss content and increases group awareness; 2) an evaluation mechanism based on Barros and Felisa Verdejo (2000), which provides a tool for the instructor to monitor and evaluate students' performance; and 3) a project management tool, which increases awareness of the status of each part of the writing project. These mechanisms are only a subset of those that we think are important. There is still much room for improving the functionality and features of wikis to foster the collaborative aspects of learning. We do not claim to have exhausted all possible mechanism designs to support collaborative writing; however, we believe that our proposed design provides an interesting perspective on how discovering problems in pedagogies leads to the design of innovative IT artifacts. We hope that other researchers will find our designs useful in developing extensions for MediaWiki to fulfill the goals of mandatory collaborative writing.

\section{References}

Alavi, M. (1994). Computer-mediated collaborative learning: An empirical evaluation. MIS Quarterly, $18(2), 150-174$.

Bandura, A., \& Cervone, D. (1983). Self-evaluation and self-efficacy mechanisms governing motivational effects of goal systems. Journal of Personality and Social Psychology, 45, 1017-1028. 
Barrett, D. J. (2009). MediaWiki. Beijing: O'Reilly.

Barros, B., \& Felisa Verdejo, M. (2000). Analysing student interaction processes in order to improve collaboration: The DEGREE approach. International Journal of Artificial Intelligence in Education, 11, 221-241.

Bean, L., \& Hott, D. D. (2005, July/August). Wiki: A speedy new tool to manage projects. Journal of Corporate Accounting \& Finance, 16(5), 3-8.

Beck, E., \& Bellotti, V. (1993). Informed opportunisms as strategy: Supporting coordination in distributed collaborative writing. In C. S. G. De Michelis, \& K. Schmidt (Eds.), Proceedings of the European Conference of Computer-Supported Cooperative Work (pp. 233-248). Dordrecht: Kluwer.

Bouton, C., \& Garth, R. Y. (1983). Learning in groups. New directions for teaching and learning series (Vol. 14). San Francisco: Jossey-Bass.

Bruner, J. S. (1996). The culture of education. Cambridge: Harvard University Press.

Cunningham, W. (2006). Design principles of Wiki: How can so little do so much?. Retrieved February 20, 2008, from http://c2.com/doc/wikisym/WikiSym2006.pdf

De Pedro, X., Rieradevall, M., López, P., Sant, D., Piñol, J., Núñez, L., et al. (2006). Writing documents collaboratively in higher education using traditional vs. wiki methodology (I): Qualitative results from a 2-year project study. Proceedings of the 4th International Congress of University Teaching and Innovation, Barcelona, Spain. Retrieved February 14, 2008 from http://uniwiki.ourproject.org/Article+Wikis

Dewey, J. (1910). How We Think. Boston: D. C. Heath.

Dewey, J. (1916). Democracy and education. New York: The Free Press.

Forte, A. (2006). Connecting the design of collaborative writing tools with writers' practices and epistemological beliefs. Doctoral Colloquium, Computer-Supported Collaborative Work (CSCW), Banff, Canada. Retrieved October 15, 2008 from http://www.cc.gatech.edu/ aforte/ForteCSCWDocConsortium.pdf

Forte, A., \& Bruckman, A. (2007). Constructing text: Wiki as a toolkit for (collaborative?) learning. Proceedings of International Symposium on Wikis (WikiSym), Montréal, Canada, 31-42. Retrieved October 15, 2008 from http://www.cc.gatech.edu/ aforte/ForteBruckmanConstructingText.pdf

Harasim, L. (1990). On-Line education: Perspectives on a new medium. New York: Praeger/Greenwood.

Hevner, A. R., March, S. T., Park, J., \& Ram, S. (2004). Design Research in Information Systems Research. MIS Quarterly, 28(1), 75-105.

Hiltz, S. R., Coppola, N., Rotter, N., \& Turoff, M. (1999). Measuring the importance of collaborative learning for the effectiveness of ALN: A multi-measure, multi-method approach. In J. Bourne (Ed.), Proceedings of the 1999 Sloan Summer Workshop on Asynchronous Learning Networks: Online education Vol. 1. Learning effectiveness and faculty satisfaction. (pp. 101-120). Sloan-C.

The Institute for the Future of the Book. (n.d.). Gamer theory. Retrieved February 14, 2009, from http://www.futureofthebook.org/gamertheory/?cat $=5$

Kriplean, T., Beschastnikh, I., McDonald, D. W., \& Golder, S. A. (2007). Community, consensus, coercion, control: $\mathrm{cs}^{*} \mathrm{w}$ or how policy mediates mass participation. Proceedings of the 2007 International ACM Conference on Supporting Group Work, 167-176.

Liccardi, I. (2007). CAWS: Improving Users' Awareness in collaborative authoring activities. Proceedings of the Conference on Supporting Group Work Group '07 Doctoral Consortium papers, Sanibel Island, Florida.

Liccardi, I., Davis, H. C., \& White, S. (2007). CAWS: A wiki system to improve workspace awareness to advance effectiveness of co-authoring activities. Proceedings of the Conference on Human Factors in Computing Systems CHI '07, San Jose, California, USA, 2555 - 2560. 
Liccardi, I., Davis, H. C., \& White, S. (2008a). CAWS: An awareness based wiki system to improve team collaboration. Proceedings of the eighth IEEE International Conference on Advanced Learning Technologies, Santander, Cantabria, Spain, 265-267.

Liccardi, I., Davis, H. C., \& White, S. (2008b). CAWS: Visualizing awareness to improve the effectiveness of co-authoring activities. Special Issue of Collaborative Computing in IEEE Distributed Systems Online. (In Press)

McCarthy, J., Miles, V., \& Monk, A. (1991). An experimental study of common ground in text-based communication. Proceedings of the SIGCHI conference on Human factors in computing systems: Reaching through technology, New Orleans, Louisiana, USA, 209-215.

Parker, K. R., \& Chao, J. T. (2007). Wiki as a teaching tool. Interdisciplinary Journal of Knowledge and Learning Objects, 3, 57-72. Retrieved from http://ijello.org/Volume3/IJKLOv3p057-072Parker284.pdf

Peffers, K., Tuunanen, T., Rothenberger, M. A., \& Chatterjee, S. (2007). A design science research methodology for information systems research. Journal of Management Information Systems, 24(3), 45-77.

Posner, I. R., \& Baecker, R. M. (1992). How people write together. Proceedings of the Twenty-Fifth Annual Hawaii International Conference on System Sciences, Kauai, Hawaii, USA, 4, 127-138.

Raman, M., Ryan, T., \& Olfman, L. (2005). Designing knowledge management systems for teaching and learning with wiki technology. Journal of Information Systems Education, 16(3), 311-320.

Richardson, W. (2006). Blogs, Wikis, podcasts, and other powerful Web tools for classrooms. Thousand Oaks, CA: Corwin Press.

Schulz, K. H., \& Ludlow, D. K. (1996, July). Incorporating group writing instruction in engineering courses. Journal of Engineering Education, 85(3), 227-232.

Suh, B., Chi, E. H., Kittur, A., \& Pendleton, B. A. (2008). Lifting the veil: Improving accountability and social transparency in Wikipedia with WikiDashboard. Proceeding of the Twenty-Sixth Annual SIGCHI Conference on Human Factors in Computing Systems, Florence, Italy, 1037-1040.

Suh, H.-J., \& Lee, S.-W. (2006). Collaborative learning agent for promoting group interaction. Electronics Telecommunications Research Institute (ETRI) Journal, 28(4), 461-474.

Tammaro, S. G., Mosier, J. N., Goodwin, N. C., \& Spitz, G. (1997). Collaborative writing is hard to support: A field study of collaborative writing. Computer Supported Cooperative Work: The Journal of Collaborative Computing, 6, 19-51.

University of Delaware. (2008, May 23). Wiki in higher education. Newark, DE: University of Delaware, IT-User Services. Retrieved October 15, 2008, from http://udel.edu/ mathieu/wiki/resources/2008-523 Wikis in Higher_Education UD.pdf

Wegerif, R. (1998). The social dimension of asynchronous learning networks. Journal of Asynchronous Learning Networks, 2(1), 34-49.

Weng, C., \& Gennari, J. H. (2004). Asynchronous collaborative writing through annotations. Proceedings of the 2004 ACM Conference on Computer-Supported Cooperative Work, Chicago, Illinois, USA. 578581.

Weng, C., Gennari, J. H., \& McDonald, D. W. (2004). A collaborative clinical trial protocol writing system. In M. Fieschi et al. (Eds.), Proceedings of the $11^{\text {th }}$ World Congress on Medical Informatics (pp. 1481-1486). Amsterdam: IOS Press.

Vygotsky, L. S. (1978). Mind in society. Cambridge: Harvard University Press.

Wiki Design Principles. (n.d.). Retrieved February 15, 2008, from http://c2.com/cgi/wiki?WikiDesignPrinciples 


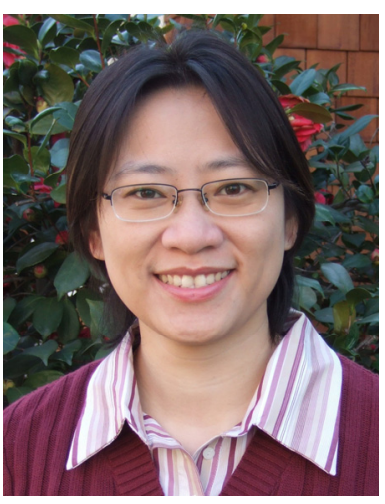

\section{Biographies}

Sumonta Kasemvilas is a $\mathrm{PhD}$ student and a research assistant in the Social Learning Software Lab ([SL]2]) at the School of Information Systems and Technology (SISAT), Claremont Graduate University (CGU). Her research interests are Web and Internet technologies, online intellectual communities, group collaboration, knowledge management, and social network analysis. Her dissertation research addresses the design and development of plug-ins for wikis to support group collaboration and knowledge sharing in the classroom setting. She is also conducting a study on an anchored discussion tool using social network analysis.

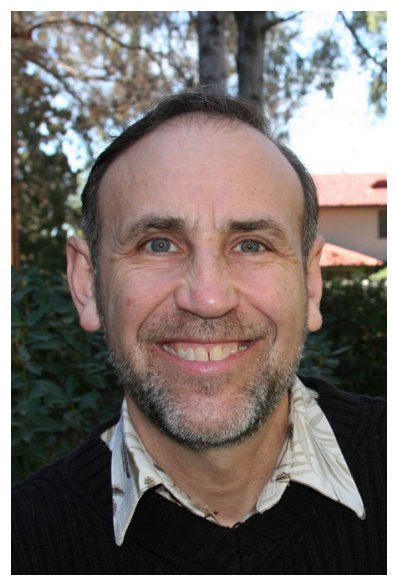

Lorne Olfman is a Professor in the School of Information Systems and Technology and Fletcher Jones Chair in Technology Management at Claremont Graduate University (CGU). He came to Claremont in 1987 after graduating with a $\mathrm{PhD}$ in Business (Management Information Systems) from Indiana University. Lorne's research interests include: how software can be learned and used in organizations, the impact of computer-based systems on knowledge management, and the design and adoption of systems used for group work. Along with Terry Ryan, Lorne co-directs the Social Learning Software Lab ([SL]2). A key component of Lorne's teaching is his involvement with doctoral students; he has supervised 45 students to completion. Lorne is an active member of the Information Systems community. 